VOL. 57 (1998) [403-408]

\title{
FINITE-DIMENSIONAL SIMPLE MODULES OVER QUANTISED WEYL ALGEBRAS
}

\author{
NOBUYUKI FUKUDA
}

We classify finite-dimensional simple modules over quantised $n$-th Weyl algebras $A_{n}^{\bar{q}, \Lambda}$ over an algebraically closed field under a certain condition on the parameters.

\section{INTRODUCTION}

Several authors have proposed various algebras as $q$-analogues to the Weyl algebras. See, for example, $[\mathbf{3}, \mathbf{1}, \mathbf{5}, \mathbf{2}]$. Since the $n$-th Weyl algebra is the algebra of differential operators on the $n$-dimensional affine space, these $q$-analogues to the $n$-th Weyl algebara have been regarded as the algebras of quantised differential operators on $n$-dimensional quantum affine spaces. In this paper we deal with the quantised Weyl algebras $A_{n}^{\bar{q}, \Lambda}$ studied in $[\mathbf{1}, \mathbf{5}]$ et cetera.

Although the Weyl algebras (over a field of characteristic 0 ) have no non-zero finitedimensional module, the quantised Weyl algebras have them. The purpose of this paper is to classify finite-dimensional simple modules over the quantised Weyl algebras $A_{n}^{\bar{q}, \Lambda}$ under a certain condition on the parameters. For this end, the classification result for $n=1$ due to Jordan [4] is crucial.

Throughout this paper, let $k$ be an algebraically closed field of arbitrary characteristic.

\section{Quantised Weyl algebras $A_{n}^{\bar{q}, \Lambda}$}

DEFINITION 1.1: ([1].) Let $\Lambda=\left(\lambda_{i j}\right)$ be an $n \times n$ matrix over the multiplicative group $k^{\times}$of $k$ such that $\lambda_{i i}=1$ for each $i$ and such that $\lambda_{i j}=\lambda_{j i}^{-1}$ for each $i, j$, and let $\bar{q}=\left(q_{1}, \cdots, q_{n}\right)$ be an $n$-tuple of elements of $k \backslash\{0,1\}$. The $n$-th quantised Weyl algebra $A_{n}^{\bar{q}, \Lambda}$ is by definition the $k$-algebra generated by $2 n$ elements $y_{1}, \cdots, y_{n}, x_{1}, \cdots, x_{n}$ with relations

$$
x_{i} x_{j}=q_{i} \lambda_{i j} x_{j} x_{i}
$$

Received 3rd September, 1997

I would like to thank Professors Y. Hirano, S. Ikehata and A. Nakajima for encouragement and valuable comments.

Copyright Clearance Centre, Inc. Serial-fee code: 0004-9729/98 \$A2.00+0.00. 


$$
\begin{aligned}
y_{i} y_{j} & =\lambda_{i j} y_{j} y_{i}, \\
x_{i} y_{j} & =\lambda_{j i} y_{j} x_{i}, \\
y_{i} x_{j} & =q_{i}^{-1} \lambda_{j i} x_{j} y_{i}, \\
x_{j} y_{j}-q_{j} y_{j} x_{j} & =1+\sum_{l=1}^{j-1}\left(q_{l}-1\right) y_{l} x_{l}, \\
\left(x_{1} y_{1}-\right. & \left.q_{1} y_{1} x_{1}=1\right),
\end{aligned}
$$

where $1 \leqslant i<j \leqslant n$. When $n=1, \Lambda=(1)$ and $\bar{q}=\left(q_{1}\right), A_{1}^{\bar{q}, \Lambda}$ is abbreviated to $A_{1}^{q}$, where $q=q_{1}$.

For $1 \leqslant i \leqslant n$, let $z_{i}=1+\sum_{j=1}^{i}\left(q_{j}-1\right) y_{j} x_{j}$. These elements of $A_{n}^{\bar{q}, \Lambda}$ are called the Casimir elements, and play an important role in investigating the quantised Weyl algebras. By a direct computation we get the following result (see $[5,2.8]$ ).

LEMMA 1.3. The Casimir elements $z_{1}, \cdots, z_{n}$ of $A_{n}^{\bar{q}, \Lambda}$ satisfy the following relations:

$$
z_{j} y_{i}=\left\{\begin{array}{ll}
y_{i} z_{j} & \text { if } j<i, \\
q_{i} y_{i} z_{j} & \text { if } j \geqslant i,
\end{array} \quad z_{j} x_{i}=\left\{\begin{array}{ll}
x_{i} z_{j} & \text { if } j<i, \\
q_{i}^{-1} x_{i} z_{j} & \text { if } j \geqslant i,
\end{array} \quad z_{i} z_{j}=z_{j} z_{i}\right.\right.
$$

for $1 \leqslant i, j \leqslant n$.

For $1 \leqslant i \leqslant n$, let $\mathcal{Y}_{i}=\left\{y_{i}^{j}\right\}_{j \geqslant 1}, \mathcal{X}_{i}=\left\{x_{i}^{j}\right\}_{j \geqslant 1}$ and $\mathcal{Z}_{i}=\left\{z_{i}^{j}\right\}_{j \geqslant 1}$ in $A_{n}^{\bar{q}, \Lambda}$. Note that $\mathcal{Y}_{1}, \cdots, \mathcal{Y}_{n}, \mathcal{X}_{1}, \cdots, \mathcal{X}_{n}, \mathcal{Z}_{1}, \cdots, \mathcal{Z}_{n}$ and the product $\mathcal{Z}=\mathcal{Z}_{1} \cdots \mathcal{Z}_{n}$ are Ore sets in $A_{n}^{\bar{q}, \Lambda}$. We denote by $B_{n}^{\bar{q}, \Lambda}$ the localisation of $A_{n}^{\bar{q}, \Lambda}$ at $\mathcal{Z}$. It is proved in [5, Theorem 3.2] that, if no $q_{i}$ is a root of unity, then $B_{n}^{\bar{q}, \Lambda}$ is simple, so that $B_{n}^{\bar{q}, \Lambda}$ has no non-zero finite-dimensional module, since $B_{n}^{\bar{q}, \Lambda}$ is infinite-dimensional over $k$.

\section{Finite-dimensional Simple MOdUles OVER $A_{n}^{\bar{q}, \Lambda}$}

LemMA 2.1. Fix $1 \leqslant i \leqslant n$. Suppose that $q_{i}$ is not a root of unity. Let $V$ be a finite-dimensional $A_{n}^{\bar{q}, \Lambda}$-module. If $V$ is $\mathcal{Z}_{j}$-torsion-free for some $j \geqslant i$, then the endomorphisms induced by $x_{i}$ and $y_{i}$ on $V$ are nilpotent.

PROOF: If $x_{i}$ does not act on $V$ as a nilpotent endomorphism, there is a non-zero eigenvalue $\mu \in k$ for the action of $x_{i}$ on $V$. Let $v \in V$ be a eigenvector with the eigenvalue $\mu$. It follows from the assumption that $v z_{j}^{m} \neq 0$ for each $m \geqslant 0$. Hence by Lemma 1.3 one sees that $x_{i}$ has infinitely many eigenvalues $\left\{q_{i}^{-m} \mu\right\}_{m \geqslant 0}$ on $V$, which contradicts the fact that $V$ is of finite dimension. The same argument is valid for $y_{i}$.

In [4] Jordan classified finite-dimensional simple modules over certain iterated skew polynomial rings, which include the first quantised Weyl algebra $A_{1}^{q}$. We shall describe the classification result for $A_{1}^{q}$ when $q$ is not a root of unity. 
Definition 2.2: [4] Let $q \in k \backslash\{0,1\}, R=A_{1}^{q}$. For $\mu \in k^{\times}$, denote by $C(\mu)$ the right $R$-module

$$
R /(z R+(y-\mu) R)
$$

If we denote by $v$ the image of 1 via the canonical surjection $R \rightarrow C(\mu)$, one sees that

$$
C(\mu)=k v, \quad v y=\mu v, \quad v x=\frac{1}{\mu(1-q)} v .
$$

(In [4] the $R$-module $C(\mu)$ is denoted by $C(0, \mu)$.)

Proposition 2.3. ([4].) Suppose that $q$ is not a root of unity. Then every finite-dimensional simple module over $A_{1}^{q}$ is isomorphic to $C(\mu)$ for some $\mu \in k^{\times}$.

Next we consider finite-dimensional simple modules over $n$-th quantised Weyl algebras $A_{n}^{\bar{q}, \Lambda}$ for $n \geqslant 2$.

Lemma 2.4. Suppose that $q_{1}$ is not a root of unity. Let $V$ be a finite-dimensional simple module over $A_{n}^{\bar{q}, \Lambda}$.

(i) Both $x_{1} y_{1}$ and $y_{1} x_{1}$ act on $V$ as the scalar $\left(1-q_{1}\right)^{-1}$,

(ii) $V x_{i} y_{i}=V y_{i} x_{i}=0$ for $2 \leqslant i \leqslant n$.

Proof: Since $A_{1}^{q_{1}}$ is a subalgebra of $A_{n}^{\bar{q}, \Lambda}, V$ contains $C(\mu)$ for some $\mu \in k^{\times}$by Proposition 2.3. Thus there is a non-zero element $v \in V$ such that $v y_{1}=\mu v, v x_{1}=$ $\left(\mu\left(1-q_{1}\right)\right)^{-1} v$. In particular it follows that $y_{1}$ is not nilpotent on $V$, so that by Lemma 2.1, $V$ is $\mathcal{Z}_{j}$-torsion, equivalently $V z_{j}=0$ for $j=1, \cdots, n$. By using the relations (1.2), the lemma follows.

COROLlaRY 2.5. If $q_{1}$ is not a root of unity, then there exists no non-zero finite-dimensional module over $B_{n}^{\bar{q}, \Lambda}$.

Proof: Suppose that there is a finite-dimensional non-zero $B_{n}^{\bar{q}, \Lambda}$-module $V$. Since $z_{1}$ is a unit in $B_{n}^{\bar{q}, \Lambda}, V$ is $\mathcal{Z}_{1}$-torsion-free, so that $x_{1}$ and $y_{1}$ act nilpotently on $V$ by Lemma 2.1. On the other hand, it follows from Lemma 2.4(i) that $V$ contains a non- $\mathcal{X}_{1}$-torsion element, which is a contradiction.

From relation (1.2) and Lemma 2.4, we get the following lemma in the same way as the proof of [6, Lemma 4].

Lemma 2.6. Suppose that $q_{1}$ is not a root of unity. Let $V$ be a finite-dimensional simple module over $A_{n}^{\bar{q}, \Lambda}$. Then the endomorphisms on $V$ induced by $x_{1}, \cdots, x_{n}, y_{1}, \cdots, y_{n}$ are diagonalisable.

Lemma 2.7. Suppose that $q_{1}$ is not a root of unity. Let $V$ be a finite-dimensional simple module over $A_{n}^{\bar{q}, \Lambda}$. Fix $1 \leqslant i<j \leqslant n$.

(i) If $\lambda_{i j}^{m} \neq 1$ for any positive integer $m \leqslant \operatorname{dim} V$, then $V y_{i}=V x_{i}=0$ or $V y_{j}=0$. 
(ii) If $\left(q_{i} \lambda_{i j}\right)^{m} \neq 1$ for any positive integers $m \leqslant \operatorname{dim} V$, then $V y_{i}=V x_{i}=0$ or $V x_{j}=0$.

Proof: Let $W$ be a $A_{n}^{\bar{q}, \Lambda}$-module. For $r \in A_{n}^{\bar{q}, \Lambda}, \mu \in k$, write

$$
W(r ; \mu)=\{w \in W \mid w r=\mu w\}
$$

the eigenspace of $r$ corresponding to the eigenvalue $\mu$. By a direct computation using relations (1.2) it follows that for $m \geqslant 0$

$$
\begin{array}{lll}
W\left(x_{i} ; \mu\right) x_{j}^{m} \subset W\left(x_{i} ;\left(q_{i} \lambda_{i j}\right)^{-m} \mu\right), & W\left(y_{i} ; \mu\right) x_{j}^{m} \subset W\left(y_{i} ;\left(q_{i} \lambda_{i j}\right)^{m} \mu\right), \\
W\left(x_{i} ; \mu\right) y_{j}^{m} \subset W\left(x_{i} ; \lambda_{i j}^{m} \mu\right), & W\left(y_{i} ; \mu\right) y_{j}^{m} \subset W\left(y_{i} ; \lambda_{j i}^{m} \mu\right),
\end{array}
$$

where $i<j$. By taking $W$ to be $V$ in the above, the lemma follows immediately.

Put $R=A_{n}^{\bar{q}, \Lambda}$. For an $n$-tuple $\boldsymbol{\mu}=\left(\mu_{1}, \cdots, \mu_{n}\right)$ of elements of $k$ with $\mu_{1} \neq 0$, denote by $D(\boldsymbol{\mu})$ (respectively $D^{\dagger}(\boldsymbol{\mu})$ ) the right $R$-module

$$
\begin{aligned}
& R /\left(\sum_{i=1}^{n}\left(y_{i}-\mu_{i}\right) R+\left(x_{1}-\left(\mu_{1}\left(1-q_{1}\right)\right)^{-1}\right) R+\sum_{i=2}^{n} x_{i} R\right) \\
& \text { (respectively } \left.R /\left(\left(y_{1}-\mu_{1}\right) R+\sum_{i=2}^{n} y_{i} R+\sum_{i=1}^{n}\left(x_{i}-\mu_{i}\right) R\right)\right) .
\end{aligned}
$$

These modules are of dimension $\leqslant 1$. Clearly $D\left(\mu_{1}, 0, \cdots, 0\right)=D^{\dagger}\left(\mu_{1}, 0, \cdots, 0\right)$ is 1-dimensional. From Lemma 2.4 and Lemma 2.7 we deduce easily the following.

Corollary 2.8. Suppose that $q_{1}$ is not a root of unity. If neither $\lambda_{1 j}$ nor $q_{1} \lambda_{1 j}$ is a root of unity for each $j \geqslant 2$, then every finite-dimensional simple module over $A_{n}^{\bar{q}, \Lambda}$ is isomorphic to $D(\mu, 0, \cdots, 0)$ for some $\mu \in k^{\times}$.

Corollary 2.9. Suppose that $q_{1}$ is not a root of unity. If $\lambda_{i j}=1$ for all $i, j$, then every finite-dimensional simple module over $A_{n}^{\bar{q}, \Lambda}$ is isomorphic to $D(\mu)$ for some $\mu \in k^{n}$ with $\mu_{1} \neq 0$.

PROOF: Since $y_{1}, \cdots, y_{n}, x_{1}$ commute with each other, the endomorphism induced by $y_{1}, \cdots, y_{n}, x_{1}$ on $V$ are simultaneously diagonalisable by Lemma 2.6 . Then the result follows easily.

Finally we shall consider the case when $n=2$.

We say that $\mu \in k^{\times}$is a root of unity of order $m$ if $m$ is the least positive integer such that $\mu^{m}=1$.

Put $R=A_{2}^{\bar{q}, \Lambda}, \lambda=\lambda_{12}$. For $\mu, \alpha \in k^{\times}$and a positive integer $m$, we denote by $E(\mu, m, \alpha)$ (respectively $E^{\dagger}(\mu, m, \alpha)$ ) the right $R$-module

$$
\begin{aligned}
& R /\left(\left(y_{1}-\mu\right) R+\left(x_{1}-\left(\mu\left(1-q_{1}\right)\right)^{-1}\right) R+\left(y_{2}^{m}-\alpha\right) R+x_{2} R\right) \\
& \left(\text { respectively } R /\left(\left(y_{1}-\mu\right) R+\left(x_{1}-\left(\mu\left(1-q_{1}\right)\right)^{-1}\right) R+y_{2} R+\left(x_{2}^{m}-\alpha\right) R\right)\right) .
\end{aligned}
$$


Note that $E(\mu, 1, \alpha)=D(\mu, \alpha), E^{\dagger}(\mu, 1, \alpha)=D^{\dagger}(\mu, \alpha)$. It is easy to see that $E(\mu, m, \alpha)$ (respectively $E^{\dagger}(\mu, m, \alpha)$ ) is simple if and only if $\lambda=1$ (respectively $\lambda=q_{1}^{-1}$ ), $\alpha=0$ or $\lambda$ (respectively $q_{1} \lambda$ ) is a root of unity of order $m$. We remark that $E^{(\dagger)}(\mu, m, 0) \cong$ $E^{(\dagger)}(\mu, 1,0)$ for $m \geqslant 1$. For $\mu, \mu^{\prime}, \alpha, \alpha^{\prime} \in k^{\times}$, if $\lambda$ (respectively $q_{1} \lambda$ ) is a root of unity of order $m \geqslant 2$, then the simple $R$-module $E^{(\dagger)}(\mu, m, \alpha)$ is isomorphic to $E^{(\dagger)}\left(\mu^{\prime}, m, \alpha^{\prime}\right)$ if and only if $\alpha=\alpha^{\prime}$ and $\mu^{\prime}=\lambda^{d} \mu$ (respectively $\mu^{\prime}=\left(q_{1} \lambda\right)^{d} \mu$ ) for some non-negative integer $d \leqslant m-1$.

TheOREM 2.10. Suppose that $q_{1}$ is not a root of unity. Put $\lambda=\lambda_{12}$.

(i) If neither $\lambda$ nor $q_{1} \lambda$ is a root of unity, then every finite-dimensional simple module over $A_{2}^{\bar{q}, \Lambda}$ is isomorphic to $E(\mu, 1,0)\left(=E^{\dagger}(\mu, 1,0)\right)$ for some $\mu \epsilon$ $k^{\times}$.

(ii) If $\lambda$ is a root of unity of order $m$, then every finite-dimensional simple module over $A_{2}^{\bar{q}, \Lambda}$ is isomorphic to either $E(\mu, 1,0)$ for some $\mu \in k^{\times}$or $E(\mu, m, \alpha)$ for some $\mu, \alpha \in k^{\times}$.

(iii) If $q_{1} \lambda$ is a root of unity of order $m$, then every finite-dimensional simple module over $A_{2}^{\bar{q}, \Lambda}$ is isomorphic to either $E^{\dagger}(\mu, 1,0)$ for some $\mu \in k^{\times}$or $E^{\dagger}(\mu, m, \alpha)$ for some $\mu, \alpha \in k^{\times}$.

ProOF:

(i) This is a special case of Corollary 2.8.

(ii) Put $R=A_{2}^{\bar{q}, \Lambda}$. Let $V$ be a finite-dimensional simple $R$-module. Since $q_{1} \lambda$ is not a root of unity, it follows from Lemma 2.7(ii) that $V x_{2}=0$. Suppose that $V$ is not of the form $E(\mu, 1,0)$. In particular, $V y_{1} \neq 0$ by Lemma 2.5 . Thus it suffices to show that $y_{2}^{m}$ acts on $V$ as a non-zero scalar $\alpha$. Note that $V$ is a simple module over $S=R /\left(x_{i} y_{i}-y_{i} x_{i} \mid 1 \leqslant i \leqslant n\right)$ by Lemma 2.5. From relations (1.2), the image of $y_{2}^{m}$ in $S$ is contained in the centre of $S$, which shows the above claim.

(iii) Similar to (ii).

REMARK 2.11. For arbitrary parameters $\bar{q}$ and $\Lambda$, no finite-dimensional module over the quantised Weyl algebra $A_{n}^{\bar{q}, \Lambda}$ is semisimple. For right $A_{n}^{\bar{q}, \Lambda}$-modules $V$ and $W$, we denote by $\operatorname{Ext}(V, W)$ the group of all equivalence classes of extensions of $W$ by $V$. This additive group $\operatorname{Ext}(V, W)$ is naturally a $k$-vector space. One can directly see that, for $\mu \in k^{\times}$

$$
\begin{array}{ll}
\operatorname{dim}_{k} \operatorname{Ext}(C(\mu), C(\mu))=1 & \text { (when } n=1), \\
\operatorname{dim}_{k} \operatorname{Ext}(D(\mu, 0, \cdots, 0), D(\mu, 0, \cdots, 0))=r & (\text { when } n \geqslant 2),
\end{array}
$$

where $r$ is the number of $i$ such that $\lambda_{i 1}=1$ or $q_{1}$. In the case when $n=1$, moreover, it is easy to see that, for $\mu, \mu^{\prime} \in k^{\times}$such that $\mu^{\prime} \neq \mu$ and $\mu^{\prime} \neq q \mu$,

$$
\operatorname{dim}_{k} \operatorname{Ext}(C(\mu), C(q \mu))=1, \quad \operatorname{dim}_{k} \operatorname{Ext}\left(C(\mu), C\left(\mu^{\prime}\right)\right)=0 .
$$




\section{REFERENCES}

[1] J. Alev and F. Dumas, 'Sur le corps des fractions de certaines algèbres quantique', $J$. Algebra 170 (1994), 229-265.

[2] A. Giaquinto and J.J. Zhang, 'Quantum Weyl algebras', J. Algebra 176 (1995), 861-881.

[3] K.R. Goodearl, 'Prime ideals in skew polynomial rings and quantized Weyl algebras', $J$. Algebra 150 (1992), 324-377.

[4] D.A. Jordan, 'Finite-dimensional simple modules over certain iterated skew polynomial rings', J. Pure Appl. Algebra 98 (1995), 45-55.

[5] D.A. Jordan, 'A simple localization of quantized Weyl algebra', J. Algebra 174 (1995), 267-281.

[6] M. Rosso, 'Finite dimensional representations of the quantum analog of the enveloping algebra of a complex simple Lie algebra', Commun. Math. Phys. 117 (1988), 581-593.

Department of Mathematics

Okayama University

Okayama 700

Japan

e-mail: fukuda@math.okayama-u.ac.jp 